\title{
Restriction theorems for the Fourier transform to homogeneous polynomial surfaces in $\mathbb{R}^{3}$
}

by

\author{
E. Ferreyra, T. Godoy and M. Urciuolo (Córdoba)
}

\begin{abstract}
Let $\varphi: \mathbb{R}^{2} \rightarrow \mathbb{R}$ be a homogeneous polynomial function of degree $m \geq 2$, let $\Sigma=\{(x, \varphi(x)):|x| \leq 1\}$ and let $\sigma$ be the Borel measure on $\Sigma$ defined by $\sigma(A)=$ $\int_{B} \chi_{A}(x, \varphi(x)) d x$ where $B$ is the unit open ball in $\mathbb{R}^{2}$ and $d x$ denotes the Lebesgue measure on $\mathbb{R}^{2}$. We show that the composition of the Fourier transform in $\mathbb{R}^{3}$ followed by restriction to $\Sigma$ defines a bounded operator from $L^{p}\left(\mathbb{R}^{3}\right)$ to $L^{q}(\Sigma, d \sigma)$ for certain $p, q$. For $m \geq 6$ the results are sharp except for some border points.
\end{abstract}

1. Introduction. Let $\varphi: \mathbb{R}^{n} \rightarrow \mathbb{R}$ be a smooth enough function, let $B$ be the open unit ball in $\mathbb{R}^{n}$ and let $\Sigma=\{(x, \varphi(x)): x \in B\}$. For $f \in S\left(\mathbb{R}^{n+1}\right)$, let $\mathcal{R} f: \Sigma \rightarrow \mathbb{C}$ be defined by $(\mathcal{R} f)(x, \varphi(x))=\widehat{f}(x, \varphi(x))$, $x \in B$, where $\widehat{f}$ denotes the usual Fourier transform of $f$ defined by $\widehat{f}(\xi)=$ $\int f(u) e^{-i\langle u, \xi\rangle} d u$. Let $\sigma$ be the Borel measure on $\Sigma$ defined by $\sigma(A)=$ $\int_{B} \chi_{A}(x, \varphi(x)) d x$ and let $E$ be the type set for the operator $\mathcal{R}$, i.e. the set of pairs $(1 / p, 1 / q) \in[0,1] \times[0,1]$ such that $\|\widehat{f}\|_{L^{q}(\Sigma)} \leq c\|f\|_{L^{p}\left(\mathbb{R}^{n+1}\right)}$ for some $c>0$ and all $f \in S\left(\mathbb{R}^{n+1}\right)$, where the spaces $L^{p}\left(\mathbb{R}^{n+1}\right)$ and $L^{q}(\Sigma)$ are taken with respect to the Lebesgue measure in $\mathbb{R}^{n+1}$ and the measure $\sigma$ respectively.

The $L^{p}\left(\mathbb{R}^{n+1}\right)-L^{q}(\Sigma)$ boundedness properties of the restriction operator $\mathcal{R}$ have been widely studied. It is well known that for $\Sigma$ as above, if $(1 / p, 1 / q) \in E$ then

$$
\frac{1}{q} \geq-\frac{n+2}{n} \frac{1}{p}+\frac{n+2}{n} .
$$

In [10], it is proved, for the case where $\varphi$ is a nondegenerate quadratic form in $\mathbb{R}^{n+1}$, that $(1 / p, 1 / 2) \in E$ if $(n+4) /(2 n+4) \leq 1 / p \leq 1$, and the method given there provides a general tool to obtain, from suitable estimates for $\widehat{\sigma}$, $L^{p}\left(\mathbb{R}^{n+1}\right)-L^{2}(\Sigma)$ estimates for $\mathcal{R}$. Moreover, a general theorem, due to Stein,

2000 Mathematics Subject Classification: Primary 42B10, 26D10.

Key words and phrases: restriction theorems, Fourier transform.

Research partially supported by Agencia Córdoba Ciencia, CONICET and SecytUNC. 
holds for smooth enough hypersurfaces with never vanishing Gaussian curvature (see e.g. [8, p. 386]). There it is shown that, in this case, $(1 / p, 1 / q) \in E$ if

$$
\frac{1}{q} \geq-\frac{n+2}{n} \frac{1}{p}+\frac{n+2}{n} \text { and } \frac{n+4}{2 n+4} \leq \frac{1}{p} \leq 1 .
$$

For the case $n=1$ a restriction theorem (also under the assumption of nonvanishing curvature) is given in [3] where it is proved that, in this case, $(1 / p, 1 / q) \in E$ if $3 / 4<1 / p \leq 1$ and $1 / q \geq-3 / p+3$, and this result is sharp, i.e. the conditions on $p$ and $q$ are also necessary. Also in [1], [5], [4] and [6] restriction theorems for curves of finite type are obtained. Concerning the homogeneous case, the type set $E$ is studied in [2] for $\varphi(x)=\left(\sum_{j=1}^{n}\left|x_{j}\right|^{r}\right)^{\alpha}$. The main tools used in [2] are a dyadic decomposition of $\Sigma$ combined with Strichartz's method applied to the these dyadic pieces and interpolation techniques.

In this paper we consider the case $n=2$ and $\varphi: \mathbb{R}^{2} \rightarrow \mathbb{R}$ a homogeneous polynomial function. We study the type set $E$ following in part the approach in $[2]$.

Let us describe our results. Let $E^{\circ}$ denote the relative interior of $E$ in $[0,1] \times[0,1]$.

If $\operatorname{det} \varphi^{\prime \prime}(x) \equiv 0$ we characterize $E^{\circ}$ (see Theorem 3.3).

If $\operatorname{det} \varphi^{\prime \prime}(x)$ is not identically zero and if it vanishes somewhere on $\mathbb{R}^{2}-$ $\{0\}$, since $\varphi$ is a homogeneous polynomial function, the set of the points $x$ where $\operatorname{det} \varphi^{\prime \prime}(x)$ vanishes is a finite union of lines $L_{1}, \ldots, L_{k}$ through the origin. For a point $x_{j} \in L_{j}-\{0\}, j=1, \ldots, k$, we consider the vanishing order $\alpha_{j}$ of $\operatorname{det} \varphi^{\prime \prime}(x)$ at $x_{j}$ along a transversal direction to $L_{j}$. A simple computation using the homogeneity of $\operatorname{det} \varphi^{\prime}$ shows that $\alpha_{j}$ is independent of the point $x_{j}$ and of the transversal direction chosen. Let

$$
\widetilde{m}=\max \left\{m, \alpha_{1}+2, \ldots, \alpha_{k}+2\right\} .
$$

In this case, for $\widetilde{m} \geq 6$ we characterize $E^{\circ}$, and for $\widetilde{m}<6$ we characterize $E^{\circ} \cap((3 / 4,1] \times[0,1])$ and we prove that $(3 / 4,1 / q) \in E$ for $(\widetilde{m}+2) / 8<$ $1 / q \leq 1$ (see Theorem 3.4). These results still hold if $\operatorname{det} \varphi^{\prime \prime}(x)$ never vanishes on $\mathbb{R}^{2}-\{0\}$ provided that we define $\widetilde{m}=m$ in this case.

Finally, for every case, we give (see Theorems 4.2 and 4.4) a sharp $L^{p}\left(\mathbb{R}^{3}\right)-L^{2}(\Sigma)$ estimate for the restriction operator $\mathcal{R}$.

Acknowledgements. The authors are deeply indebted to Prof. F. Ricci for his invaluable suggestions.

\section{Preliminaries}

REMARK 2.1. Let us introduce some additional notation and state some general facts concerning restriction operators. If $V \subset \mathbb{R}^{2}$ is a measurable set 
and if $\psi: V \rightarrow \mathbb{R}$ is a continuous function, let $\Sigma_{V, \psi}, \sigma_{V, \psi}, \mathcal{R}_{V, \psi}$ be the surface, the measure and the restriction operator defined as $\Sigma, \sigma$ and $\mathcal{R}$ at the beginning of the introduction, with $n=2$, but taking now $V$ and $\psi$ instead of $B$ and $\varphi$ respectively. Finally, let $E_{V, \psi}$ be the type set for $\mathcal{R}_{V, \psi}$. Let us recall some well known facts about the operators $\mathcal{R}_{V, \psi}$.

(a) The Riesz-Thorin theorem implies that $E_{V, \psi}$ is a convex set. Moreover, for $f \in S\left(\mathbb{R}^{3}\right)$, we have

$$
\left\|\mathcal{R}_{V, \psi}\right\|_{L^{1}\left(\mathbb{R}^{3}\right), L^{\infty}\left(\Sigma_{V, \psi}\right)} \leq 1 \quad \text { and } \quad\left\|\mathcal{R}_{V, \psi}\right\|_{L^{1}\left(\mathbb{R}^{3}\right), L^{1}\left(\Sigma_{V, \psi}\right)} \leq|V|
$$

where $|V|$ denotes the Lebesgue measure of $V$. So, by the Riesz-Thorin theorem, if $|V|<\infty$ the closed segment with endpoints $(1,0)$ and $(1,1)$ is contained in $E_{V, \psi}$. In particular we get the estimate

$$
\left\|\mathcal{R}_{V, \psi}\right\|_{L^{1}\left(\mathbb{R}^{3}\right), L^{2}\left(\Sigma_{V, \psi}\right)} \leq|V|^{1 / 2}
$$

(b) If $T \in \mathrm{GL}\left(\mathbb{R}^{2}\right)$ a computation shows that $E_{T(V), \psi \circ T^{-1}}=E_{V, \psi}$. Also, $E_{V, a \psi}=E_{V, \psi}$ for $a \in \mathbb{R}-\{0\}$.

(c) Let us recall the well known homogeneity argument (see e.g. [10], [11]). If $\varphi: \mathbb{R}^{2} \rightarrow \mathbb{R}$ is a continuous and homogeneous function of degree $m$ then $E_{t V, \varphi}=E_{V, \varphi}$ for all $t>0$. Indeed, a computation gives, for $f \in S\left(\mathbb{R}^{3}\right)$ and $t>0$,

$$
\|\widehat{f}\|_{L^{q}\left(\Sigma_{t V, \varphi}\right)}=t^{2 / q-(m+2)}\left\|\left(f_{t^{-1}}\right)^{\wedge}\right\|_{L^{q}\left(\Sigma_{V, \varphi}\right)}
$$

where $f_{t}\left(v_{1}, v_{2}, v_{3}\right)=f\left(t v_{1}, t v_{2}, t^{m} v_{3}\right)$. From (2.1) it easily follows that

$$
\left\|\mathcal{R}_{t V, \varphi}\right\|_{L^{p}\left(\mathbb{R}^{3}\right), L^{q}\left(\Sigma_{t V, \varphi}\right)}=t^{2 / q+(m+2) / p-(m+2)}\left\|\mathcal{R}_{V, \varphi}\right\|_{L^{p}\left(\mathbb{R}^{3}\right), L^{q}\left(\Sigma_{V, \varphi}\right)}
$$

for all $t>0$ and so $E_{t V, \varphi}=E_{V, \varphi}$.

(d) Let $\varphi$ be as in (c), let $W=\bigcup_{k \in \mathbb{N} \cup\{0\}} 2^{-k} V$ and suppose that $(1 / p, 1 / q) \in E_{V, \varphi}$ and

$$
\frac{1}{q}>-\left(\frac{m}{2}+1\right) \frac{1}{p}+\frac{m}{2}+1 .
$$

Then $(1 / p, 1 / q) \in E_{W, \varphi}$. Indeed, since

$$
\left\|\mathcal{R}_{W, \varphi}\right\|_{L^{p}\left(\mathbb{R}^{3}\right), L^{q}\left(\Sigma_{W, \varphi}\right)}^{q} \leq \sum_{k \in \mathbb{N} \cup\{0\}}\left\|\mathcal{R}_{2^{-k} V, \varphi}\right\|_{L^{p}\left(\mathbb{R}^{3}\right), L^{q}\left(\Sigma_{2^{-k} V, \varphi}\right)}^{q}
$$

the statement follows from (2.2).

(e) Another consequence of the homogeneity argument is the following. For $\varphi$ and $W$ as in (d), since

$$
\left\|\mathcal{R}_{W, \varphi}\right\|_{L^{p}\left(\mathbb{R}^{3}\right), L^{q}\left(\Sigma_{W, \varphi}\right)} \geq\left\|\mathcal{R}_{2^{-k} V, \varphi}\right\|_{L^{p}\left(\mathbb{R}^{3}\right), L^{q}\left(\Sigma_{2^{-k} V, \varphi}\right)}
$$

for all $k \in \mathbb{N}$, from (2.2) it follows that

$$
\frac{1}{q} \geq-\left(\frac{m}{2}+1\right) \frac{1}{p}+\frac{m}{2}+1
$$

is a necessary condition in order to have $(1 / p, 1 / q) \in E_{W, \varphi}$. 
The following Lemmas 2.2 and 2.4 allow us to compute the vanishing order of det $\varphi^{\prime \prime}(x)$ along the $x_{1}$ axis for an arbitrary homogeneous polynomial function $\varphi: \mathbb{R}^{2} \rightarrow \mathbb{R}$. Let $\alpha$ be the order of $x_{2}=0$ as zero of the function $x_{2} \mapsto \operatorname{det} \varphi^{\prime \prime}\left(1, x_{2}\right)$, with the convention that $\alpha=0$ if $\operatorname{det} \varphi^{\prime \prime}(1,0) \neq 0$, and $\alpha=\infty$ if $\operatorname{det} \varphi^{\prime \prime}\left(1, x_{2}\right)$ vanishes identically (i.e., by the homogeneity of $\varphi$, if $\operatorname{det} \varphi^{\prime \prime}(x)$ vanishes identically on $\left.\mathbb{R}^{2}\right)$.

Lemma 2.2. Let $\varphi: \mathbb{R}^{2} \rightarrow \mathbb{R}$ be a homogeneous polynomial function of degree $m \geq 2$ of the form

$$
\varphi\left(x_{1}, x_{2}\right)=a_{0} x_{1}^{m}+\sum_{1 \leq l \leq m} a_{l} x_{1}^{m-l} x_{2}^{l}
$$

for some $a_{0}, \ldots, a_{m} \in \mathbb{R}$ with $a_{0} \neq 0$ and let $\alpha$ be defined as above.

(i) Suppose that

$$
\frac{a_{s}}{a_{0}}=\left(\begin{array}{c}
m \\
s
\end{array}\right) m^{-s}\left(\frac{a_{1}}{a_{0}}\right)^{s} \quad \text { for } s=1, \ldots, r
$$

with $r<m$ and that

$$
\frac{a_{r+1}}{a_{0}} \neq\left(\begin{array}{c}
m \\
r+1
\end{array}\right) m^{-r-1}\left(\frac{a_{1}}{a_{0}}\right)^{r+1} .
$$

Then $\alpha=r-1$.

(ii) If

$$
\frac{a_{s}}{a_{0}}=\left(\begin{array}{c}
m \\
s
\end{array}\right) m^{-s}\left(\frac{a_{1}}{a_{0}}\right)^{s} \quad \text { for } s=1, \ldots, m,
$$

then $\alpha=\infty$.

Proof. To prove (i), without loss of generality we can assume that $a_{0}=1$. Let $r$ be as in (i), so $1 \leq r \leq m-1$. We have $\operatorname{det} \varphi^{\prime \prime}\left(x_{1}, x_{2}\right)=A B-C^{2}$ where

$$
\begin{aligned}
A & =\sum_{0 \leq l \leq m-2}(m-l)(m-l-1) a_{l} x_{1}^{m-l-2} x_{2}^{l}, \\
B & =\sum_{0 \leq j \leq m-2}(j+2)(j+1) a_{j+2} x_{1}^{m-j-2} x_{2}^{j}, \\
C & =\sum_{l=0}^{m-2}(l+1)(m-l-1) a_{l+1} x_{1}^{m-l-2} x_{2}^{l} .
\end{aligned}
$$

A computation of $A B-C^{2}$ gives

$$
\begin{aligned}
& \operatorname{det} \varphi^{\prime \prime}\left(x_{1}, x_{2}\right) \\
& =\sum_{0 \leq i \leq 2 m-4} \sum_{l+j=i}\left[(m-l)(m-l-1)(j+2)(j+1) a_{l} a_{j+2}\right. \\
& \left.\quad-(l+1)(m-l-1)(m-j-1)(j+1) a_{l+1} a_{j+1}\right] x_{1}^{2 m-4-i} x_{2}^{i} .
\end{aligned}
$$


For $0 \leq i \leq 2 m-4$, let $c_{i} x_{1}^{2 m-4-i}$ be the coefficient of $x_{2}^{i}$ in $\operatorname{det} \varphi^{\prime \prime}\left(x_{1}, x_{2}\right)$. For $s=1, \ldots, r-1$ we have

$$
\begin{aligned}
c_{s}= & \sum_{l+j=s}(m-l)(m-l-1)(j+2)(j+1) a_{l} a_{j+2} \\
& -\sum_{l+j=s}(l+1)(m-l-1)(m-j-1)(j+1) a_{l+1} a_{j+1} .
\end{aligned}
$$

Thus (recalling that $a_{0}=1$ ) the hypothesis of (i) gives

$$
\begin{aligned}
c_{s} & =m(m-1)(s+2)(s+1) a_{s+2} \\
& +\sum_{l+j=s, l \neq 0}(m-l)(m-l-1)(j+2)(j+1)\left(\begin{array}{c}
m \\
l
\end{array}\right)\left(\begin{array}{c}
m \\
j+2
\end{array}\right) m^{-s-2} a_{1}^{s+2} \\
& -\sum_{l+j=s}(l+1)(m-l-1)(m-j-1)(j+1)\left(\begin{array}{c}
m \\
l+1
\end{array}\right)\left(\begin{array}{c}
m \\
j+1
\end{array}\right) m^{-s-2} a_{1}^{s+2} .
\end{aligned}
$$

Since

$$
\begin{aligned}
(l+1)(m-l-1)(m-j-1)(j+1)\left(\begin{array}{c}
m \\
l+1
\end{array}\right)\left(\begin{array}{c}
m \\
j+1
\end{array}\right) & \\
= & (m-l)(m-l-1)(j+2)(j+1)\left(\begin{array}{c}
m \\
l
\end{array}\right)\left(\begin{array}{c}
m \\
j+2
\end{array}\right)
\end{aligned}
$$

we get

$$
c_{s}=m(m-1)(s+1)(s+2)\left[a_{s+2}-\left(\begin{array}{c}
m \\
s+2
\end{array}\right) m^{-s-2} a_{1}^{s+2}\right]
$$

and so $c_{0}=\ldots=c_{r-2}=0$ and $c_{r-1} \neq 0$, hence $\alpha=r-1$.

To see (ii) observe that if

$$
\frac{a_{s}}{a_{0}}=\left(\begin{array}{c}
m \\
s
\end{array}\right) m^{-s}\left(\frac{a_{1}}{a_{0}}\right)^{s} \quad \text { for } s=1, \ldots, m
$$

then $\varphi\left(x_{1}, x_{2}\right)=a_{0}\left(x_{1}+b x_{2}\right)^{m}$ for some $b \in \mathbb{R}$ and that in this case $\operatorname{det} \varphi^{\prime \prime}(x)$ is identically zero.

REMARK 2.3. Let $\varphi, \alpha$ be as in Lemma 2.2. Observe that this lemma implies that $\alpha<m-2$ except in the cases where $\varphi$ is either of the form $\varphi\left(x_{1}, x_{2}\right)=a_{0}\left(x_{1}+b x_{2}\right)^{m}$ or $\varphi\left(x_{1}, x_{2}\right)=a_{0}\left(x_{1}+b x_{2}\right)^{m}+b^{\prime} x_{2}^{m}$ for some $a_{0}, b, b^{\prime} \in \mathbb{R}$ with $a_{0} \neq 0, b^{\prime} \neq 0$, and that in these exceptional cases we have $\alpha=\infty$ and $\alpha=m-2$ respectively.

Lemma 2.4. Let $\varphi: \mathbb{R}^{2} \rightarrow \mathbb{R}$ be a homogeneous polynomial function of degree $m \geq 2$ given by

$$
\varphi\left(x_{1}, x_{2}\right)=\sum_{k \leq l \leq m} a_{l} x_{1}^{m-l} x_{2}^{l}
$$


for some $a_{k}, \ldots, a_{m} \in \mathbb{R}$ with $1 \leq k \leq m$, and $a_{k} \neq 0$. Let $\alpha$ be as in Lemma 2.2. Then $\alpha=2 k-2$ if $k<m$, and $\alpha=\infty$ if $k=m$.

Proof. If $k=m$, then $\varphi\left(x_{1}, x_{2}\right)=a_{m} x_{2}^{m}$, so $\operatorname{det} \varphi^{\prime \prime}\left(1, x_{2}\right)$ vanishes identically and then $\alpha=\infty$.

If $k<m$, then $\varphi\left(x_{1}, x_{2}\right)=a_{k} x_{1}^{m-k} x_{2}^{k}+x_{2}^{k+1} \psi\left(x_{1}, x_{2}\right)$ for some polynomial function $\psi$ satisfying $\psi(1,0) \neq 0$, and so

$$
\operatorname{det} \varphi^{\prime \prime}\left(x_{1}, x_{2}\right)=-k(m-1)(m-k) a_{k}^{2} x_{1}^{2 m-2 k-2} x_{2}^{2 k-2}+x_{2}^{2 k-1} \Theta\left(x_{1}, x_{2}\right)
$$

where $\Theta\left(x_{1}, x_{2}\right)$ is a polynomial function. Since $k(m-1)(m-k) \neq 0$ and $a_{k} \neq 0$, we get $\alpha=2 k-2$.

REMARK 2.5. For an arbitrary homogeneous polynomial $\varphi$ of degree $m \geq 2$, from Lemmas 2.2 and 2.4 it follows that $\operatorname{det} \varphi^{\prime \prime}\left(x_{1}, x_{2}\right) \equiv 0$ if and only if $\varphi\left(x_{1}, x_{2}\right)=\left(a x_{1}+b x_{2}\right)^{m}$ for some $a, b \in \mathbb{R}$ and all $\left(x_{1}, x_{2}\right) \in \mathbb{R}^{2}$.

REMARK 2.6. We will need the following Strichartz theorem (see [10]) whose proof relies on Stein's complex interpolation theorem which gives $L^{p}\left(\mathbb{R}^{3}\right)-L^{2}\left(\Sigma_{V, \psi}\right)$ estimates for the operator $\mathcal{R}_{V, \psi}$. Since we will need information about the size of the constants we give a sketch of its proof.

Let $V$ be a measurable subset of $\mathbb{R}^{2}$ such that $|V|>0$ and let $\psi: \mathbb{R}^{2} \rightarrow \mathbb{R}$ be a continuous function. Suppose that $\left|\left(\sigma_{V, \psi}\right)^{\wedge}(\xi)\right| \leq A\left(1+\left|\xi_{3}\right|\right)^{-\tau}$ for some $\tau>0$ and for all $\xi=\left(\xi_{1}, \xi_{2}, \xi_{3}\right) \in \mathbb{R}^{3}$. Then

$$
\left\|\mathcal{R}_{V, \psi}\right\|_{L^{p}\left(\mathbb{R}^{3}\right), L^{2}\left(\Sigma_{V, \psi}\right)} \leq c_{\tau} A^{1 /(2(1+\tau))}
$$

for $p=(2+2 \tau) /(2+\tau)$ where $c_{\tau}$ is a positive constant depending only on $\tau$. Indeed, as in $[8$, p. 381] we define the analytic family of distributions $I_{z}$ given, for $\operatorname{Re}(z)>0$, by

$$
I_{z}(t)= \begin{cases}\frac{e^{z^{2}}}{\Gamma(z)} t^{z-1} \varsigma(t) & \text { for } t>0, \\ 0 & \text { for } t \leq 0,\end{cases}
$$

where $\varsigma \in C_{\mathrm{c}}^{\infty}$ and $\varsigma(t)=1$ for $|t| \leq 1$. Also we define $J_{z}=\delta \otimes \delta \otimes I_{z}$. For $-\tau \leq \operatorname{Re}(z) \leq 1$ and $f \in S\left(\mathbb{R}^{3}\right)$, let $T_{z} f=\left(J_{z} * \sigma_{V, \psi}\right)^{\wedge} * f$. A computation shows that if $\operatorname{Re}(z)=1$, then $\left\|J_{z} * \sigma_{V, \psi}\right\|_{L^{\infty}\left(\mathbb{R}^{3}\right)} \leq c<\infty$, so

$$
\left\|T_{z} f\right\|_{L^{2}\left(\mathbb{R}^{3}\right)}=\left\|\left(T_{z} f\right)^{\wedge}\right\|_{L^{2}\left(\mathbb{R}^{3}\right)} \leq c\|\widehat{f}\|_{L^{2}\left(\mathbb{R}^{3}\right)}=c\|f\|_{L^{2}\left(\mathbb{R}^{3}\right)} .
$$

Also since $\left|\left(J_{z}\right)^{\wedge}(\xi)\right| \leq c_{\tau}\left(1+\left|\xi_{3}\right|\right)^{\tau}$ for $\operatorname{Re}(z)=-\tau$, Young's inequality gives

$$
\left\|T_{z} f\right\|_{L^{\infty}\left(\mathbb{R}^{3}\right)}=\left\|\left(\left(\sigma_{V, \psi}\right)^{\wedge}\left(J_{z}\right)^{\wedge}\right) * f\right\|_{L^{\infty}\left(\mathbb{R}^{3}\right)} \leq c_{\tau} A\|f\|_{L^{1}\left(\mathbb{R}^{3}\right)}
$$

and so Stein's complex interpolation theorem (as stated e.g. in $[9, \mathrm{Ch} . \mathrm{V}]$ ) entails that the operator $T_{0} f=\left(\sigma_{V, \psi}\right)^{\wedge} * f$ satisfies

$$
\left\|T_{0}\right\|_{L^{p}\left(\mathbb{R}^{3}\right), L^{p^{\prime}}\left(\mathbb{R}^{3}\right)} \leq c_{\tau}^{\prime} A^{1 /(1+\tau)}
$$


for $p=(2+2 \tau) /(2+\tau)$. This implies (see e.g. [8, p. 253]) that

$$
\left\|\mathcal{R}_{V, \psi}\right\|_{L^{p}\left(\mathbb{R}^{3}\right), L^{2}\left(\Sigma_{V, \psi}\right)} \leq c_{\tau}^{\prime \prime} A^{1 /(2(1+\tau))} .
$$

Lemma 2.7. Let $\varphi\left(x_{1}, x_{2}\right)=\sum_{k<l<m} a_{l} x_{1}^{m-l} x_{2}^{l}$ where $0 \leq k \leq m$ and $a_{k}, \ldots, a_{m} \in \mathbb{R}$ with $a_{k} \neq 0$, and let $\Sigma$ and $E$ be defined as in the introduction. If $(1 / p, 1 / q) \in E$ then

$$
\frac{1}{q} \geq-(k+1) \frac{1}{p}+k+1
$$

Proof. If $k=0$ the lemma follows from Remark 2.1(e). Suppose $k \neq 0$. For $0<\varepsilon<1$, let $f_{\varepsilon}$ be the characteristic function of the set $[0,1] \times$ $\left[0, \varepsilon^{-1 / k}\right] \times\left[0, \varepsilon^{-1}\right]$. Then for $x=\left(x_{1}, x_{2}\right)$,

$$
\begin{aligned}
\widehat{f}_{\varepsilon}(x, \varphi(x)) & =\int_{0}^{1 \varepsilon^{-1 / k}} \int_{0}^{\varepsilon^{-1}} \int_{0} e^{-i\left(x_{1} \xi_{1}+x_{2} \xi_{2}+\varphi\left(x_{1}, x_{2}\right) \xi_{3}\right)} d \xi_{1} d \xi_{2} d \xi_{3} \\
& =\varepsilon^{-(1+1 / k)} \int_{Q} e^{-i\left(x_{1} u_{1}+\varepsilon^{-1 / k} x_{2} u_{2}+\varepsilon^{-1} \varphi\left(x_{1}, x_{2}\right) u_{3}\right)} d u_{1} d u_{2} d u_{3}
\end{aligned}
$$

where $Q=[0,1] \times[0,1] \times[0,1]$. Let $c=\min \left(1,\left(\frac{1}{3} \sum_{k \leq l \leq m}\left|a_{l}\right|\right)^{-1 / k}\right)$ and $D_{\varepsilon}=[0,1 / 3] \times\left[0,(c / 3) \varepsilon^{1 / k}\right]$. So $\Sigma_{D_{\varepsilon}, \varphi} \subset \Sigma$ and $\left\|\widehat{f}_{\varepsilon}\right\|_{L^{q}(\Sigma)} \geq\left\|\widehat{f}_{\varepsilon}\right\|_{L^{q}\left(\Sigma_{D_{\varepsilon}, \varphi}\right)}$. Now

$$
\begin{aligned}
\varepsilon^{q+q / k} & \left\|\widehat{f}_{\varepsilon}\right\|_{L^{q}\left(\Sigma_{D_{\varepsilon}, \varphi}\right)}^{q} \\
& =\int_{D_{\varepsilon}}\left|\int_{Q} e^{-i\left(x_{1} u_{1}+\varepsilon^{-1 / k} x_{2} u_{2}+\varepsilon^{-1} \varphi\left(x_{1}, x_{2}\right) u_{3}\right)} d u_{1} d u_{2} d u_{3}\right|^{q} d x_{1} d x_{2} \\
& \geq \int_{D_{\varepsilon}}\left|\int_{Q} \cos \left(x_{1} u_{1}+\varepsilon^{-1 / k} x_{2} u_{2}+\varepsilon^{-1} \varphi\left(x_{1}, x_{2}\right) u_{3}\right) d u_{1} d u_{2} d u_{3}\right|^{q} d x_{1} d x_{2}
\end{aligned}
$$

For $\left(x_{1}, x_{2}, \varphi\left(x_{1}, x_{2}\right)\right) \in \Sigma_{D_{\varepsilon}, \varphi}$ we have

$$
\begin{aligned}
\varepsilon^{-1}\left|\varphi\left(x_{1}, x_{2}\right)\right| & \leq \varepsilon^{-1}\left(\left|a_{k} x_{1}{ }^{m-k} x_{2}^{k}\right|+\ldots+\left|a_{m} x_{2}{ }^{m}\right|\right) \\
& \leq c^{k}\left|a_{k}\right|+\left|a_{k+1}\right| c^{k+1} \varepsilon^{1 / k}+\ldots+\left|a_{m}\right| c^{m} \varepsilon^{(m-k) / k} \leq 1 / 3
\end{aligned}
$$

and so for $\left(u_{1}, u_{2}, u_{3}\right) \in Q$ we get $\left|x_{1} u_{1}+\varepsilon^{-1 / k} x_{2} u_{2}+\varepsilon^{-1} \varphi\left(x_{1}, x_{2}\right) u_{3}\right| \leq 1<$ $\pi / 3$. Thus $\varepsilon^{q+q / k}\left\|\widehat{f}_{\varepsilon}\right\|_{L^{q}\left(\Sigma_{D_{\varepsilon}, \varphi}\right)}^{q} \geq c^{\prime} \varepsilon^{1 / k}$ with $c^{\prime}$ independent of $\varepsilon$ and $f$. Now, $\left\|f_{\varepsilon}\right\|_{L^{p}\left(\mathbb{R}^{3}\right)}=\varepsilon^{-(1+1 / k) / p}$. So from the inequality $\left\|\widehat{f}_{\varepsilon}\right\|_{L^{q}(\Sigma)} \leq c\left\|f_{\varepsilon}\right\|_{L^{p}\left(\mathbb{R}^{3}\right)}$, applied with $\varepsilon$ small enough, we get

$$
-\left(1+\frac{1}{k}\right)+\frac{1}{k} \frac{1}{q} \geq-\left(1+\frac{1}{k}\right) \frac{1}{p}
$$

and the lemma follows. 
REMARK 2.8. It is known that if $\left(\int_{0}^{1}\left|\widehat{h}\left(x_{2}, x_{2}^{2}\right)\right|^{q} d x_{2}\right)^{1 / q} \leq c\|h\|_{L^{p}\left(\mathbb{R}^{2}\right)}$ for some $c>0$ and all $h \in S\left(\mathbb{R}^{2}\right.$ ) then $1 / p>3 / 4$ (see [4, Theorem 2]). This result implies the following.

Let $\varphi: \mathbb{R}^{2} \rightarrow \mathbb{R}$ be a quadratic homogeneous polynomial function such that $\operatorname{det} \varphi^{\prime \prime}(x) \equiv 0$, and let $\Sigma$ and $E$ be defined as in the introduction. If $1 \leq p, q \leq \infty$ and there exists $c>0$ such that

$$
\|\widehat{f}\|_{L^{q}(\Sigma)} \leq c\|f\|_{L^{p}\left(\mathbb{R}^{3}\right)}
$$

for all $f \in S\left(\mathbb{R}^{3}\right)$, then $1 / p>3 / 4$. Indeed, from Remark 2.5 we have $\varphi\left(x_{1}, x_{2}\right)=\left(a x_{1}+b x_{2}\right)^{2}$ for some $a, b \in \mathbb{R}$ and all $\left(x_{1}, x_{2}\right) \in \mathbb{R}^{2}$. So, from Remark 2.1(b) the problem reduces (after composing with a suitable rotation followed by a dilation) to the case $\varphi\left(x_{1}, x_{2}\right)=x_{2}^{2}$. Let $g \in S(\mathbb{R})$ be such that $\widehat{g}>0$ on $[0,1]$. For $h \in S\left(\mathbb{R}^{2}\right)$ we take $f\left(x_{1}, x_{2}, x_{3}\right)=g\left(x_{1}\right) h\left(x_{2}, x_{3}\right)$ in (2.6) to obtain

$$
\|\widehat{g}\|_{L^{q}(0,1)}\left(\int_{0}^{1}\left|\widehat{h}\left(x_{2}, x_{2}^{2}\right)\right|^{q} d x_{2}\right)^{1 / q} \leq c\|g\|_{L^{p}(\mathbb{R})}\|h\|_{L^{p}\left(\mathbb{R}^{2}\right)}
$$

and so $\left(\int_{0}^{1}\left|\widehat{h}\left(x_{2}, x_{2}^{2}\right)\right|^{q} d x_{2}\right)^{1 / q} \leq c\|h\|_{L^{p}\left(\mathbb{R}^{2}\right)}$ for some $c>0$ and all $h \in$ $S\left(\mathbb{R}^{2}\right)$.

3. $L^{p}\left(\mathbb{R}^{3}\right)-L^{q}(\Sigma)$ estimates for $\mathcal{R}$. For $\delta>0$ we set

$$
V_{\delta}=\left\{\left(x_{1}, x_{2}\right) \in \mathbb{R}^{2}:\left|x_{1}\right| \leq 1,\left|x_{2}\right| \leq \delta\left|x_{1}\right|\right\} .
$$

Lemma 3.1. Let $\varphi: \mathbb{R}^{2} \rightarrow \mathbb{R}$ be a homogeneous polynomial function such that $\operatorname{det} \varphi^{\prime \prime}(x)$ does not vanish identically, let $\alpha$ be defined as in the preliminaries and let $m^{*}=\max (m, \alpha+2)$. Let $V_{\delta}$ be defined by (3.1). Then for $\delta$ positive and small enough:

(i) if $m^{*}<6$,

$$
\frac{3}{4} \leq \frac{1}{p} \leq 1 \quad \text { and } \quad-\left(\frac{m^{*}}{2}+1\right) \frac{1}{p}+\frac{m^{*}}{2}+1<\frac{1}{q} \leq 1,
$$

then $(1 / p, 1 / q) \in E_{V_{\delta}, \varphi}$,

(ii) if $m^{*} \geq 6$ and

$$
-\left(\frac{m^{*}}{2}+1\right) \frac{1}{p}+\frac{m^{*}}{2}+1<\frac{1}{q} \leq 1,
$$

then $(1 / p, 1 / q) \in E_{V_{\delta}, \varphi}$.

Proof. From Remark 2.1(a), to prove the lemma it suffices to show that, for $\delta$ positive and small enough, the following assertions hold:

(i') If $m-2 \leq \alpha<4$ and $(\alpha+4) / 8<1 / q \leq 1$ then $(3 / 4,1 / q) \in E_{V_{\delta}, \varphi}$. 
$\in E_{V_{\delta}, \varphi}$.

(ii') If $m-2 \leq \alpha, \alpha \geq 4$ and $(\alpha+2) /(\alpha+4)<1 / p \leq 1$ then $(1 / p, 1)$

$\left(\right.$ (iii $\left.^{\prime}\right)$ If $0 \leq \alpha<m-2, m \geq 6$, and $m /(m+2)<1 / p \leq 1$ then $(1 / p, 1)$ $\in E_{V_{\delta}, \varphi}$.

(iv $\left.{ }^{\prime}\right)$ If $0 \leq \alpha<m-2, m<6$ and $(m+2) / 8<1 / q \leq 1$ then $(3 / 4,1 / q)$ $\in E_{V_{\delta}, \varphi}$.

Let $\delta_{0}>0$ be such that $\operatorname{det} \varphi^{\prime \prime}(x) \neq 0$ for all $x=\left(x_{1}, x_{2}\right) \in V_{\delta_{0}}$ with $x_{2} \neq 0$. Our assumptions imply that there exist positive constants $c_{1}, c_{2}$ such that, if $\left(x_{1}, x_{2}\right) \in V_{\delta_{0}}$ and $1 / 2 \leq\left|x_{1}\right| \leq 1$, then

$$
c_{1}\left|x_{2}\right|^{\alpha} \leq\left|\operatorname{det} \varphi^{\prime \prime}\left(x_{1}, x_{2}\right)\right| \leq c_{2}\left|x_{2}\right|^{\alpha} .
$$

For $k \in \mathbb{N} \cup\{0\}$, let

$$
Q_{k}=\left\{\left(x_{1}, x_{2}\right) \in \mathbb{R}^{2}: 1 / 2 \leq\left|x_{1}\right| \leq 1,2^{-k-1} \leq\left|x_{2}\right| \leq 2^{-k}\right\},
$$

let $\varphi_{k}: Q_{0} \rightarrow \mathbb{R}$ be defined by $\varphi_{k}\left(x_{1}, x_{2}\right)=\varphi\left(x_{1}, 2^{-k} x_{2}\right)$ and let $\sigma_{Q_{k}, \varphi}$ be defined as at the beginning of the preliminaries. A change of variable gives

$$
\left(\sigma_{Q_{k}, \varphi}\right)^{\wedge}(\xi)=2^{-k}\left(\sigma_{Q_{0}, \varphi_{k}}\right)^{\wedge}\left(\xi_{1}, 2^{-k} \xi_{2}, \xi_{3}\right) .
$$

Pick $k\left(\delta_{0}\right) \in \mathbb{N}$ such that $Q_{k\left(\delta_{0}\right)} \subset V_{\delta_{0}}$. Since

$$
\left|\operatorname{det} \varphi_{k}^{\prime \prime}\left(x_{1}, x_{2}\right)\right|=2^{-2 k}\left|\operatorname{det} \varphi^{\prime \prime}\left(x_{1}, 2^{-k} x_{2}\right)\right|,
$$

from (3.2) it follows that there exists $c_{1}>0$ such that $\left|\operatorname{det} \varphi_{k}^{\prime \prime}\left(x_{1}, x_{2}\right)\right| \geq$ $c_{1} 2^{-k(\alpha+2)}$ for all $k \geq k\left(\delta_{0}\right),\left(x_{1}, x_{2}\right) \in Q_{0}$. Then Proposition 6 on p. 344 of [8] implies that there exists a positive constant $c_{3}$ such that

$$
\left|\left(\sigma_{Q_{0}, \varphi_{k}}\right)^{\wedge}\left(\xi_{1}, 2^{-k} \xi_{2}, \xi_{3}\right)\right| \leq c_{3} 2^{k(\alpha+2) / 2}\left|\left(\xi_{1}, 2^{-k} \xi_{2}, \xi_{3}\right)\right|^{-1} \leq c_{3} 2^{k(\alpha+2) / 2}\left|\xi_{3}\right|^{-1}
$$

for all $k \geq k\left(\delta_{0}\right)$ and $\xi \in \mathbb{R}^{3}$. For these $k$, from (3.4) we obtain $\left|\left(\sigma_{Q_{k}, \varphi}\right)^{\wedge}(\xi)\right| \leq$ $c_{3} 2^{k \alpha / 2}\left|\xi_{3}\right|^{-1}$. So, Remark 2.6 implies that

$$
\|\widehat{f}\|_{L^{2}\left(\Sigma_{Q_{k}, \varphi}\right)} \leq c_{4} 2^{k \alpha / 8}\|f\|_{L^{4 / 3}\left(\mathbb{R}^{3}\right)}, \quad f \in S\left(\mathbb{R}^{3}\right)
$$

with $c_{4}$ independent of $k$ and $f$. From (3.5), Hölder's inequality gives, for $1 \leq q<2$ and $f \in S\left(\mathbb{R}^{3}\right)$,

$$
\begin{aligned}
\|\widehat{f}\|_{L^{q}\left(\Sigma_{Q_{k}, \varphi}\right)} & \leq \sigma\left(\Sigma_{Q_{k}, \varphi}\right)^{(2-q) /(2 q)}\|\widehat{f}\|_{L^{2}\left(\Sigma_{Q_{k}, \varphi}\right)} \\
& \leq c_{4} 2^{k(\alpha / 8-(2-q) /(2 q))}\|f\|_{L^{4 / 3}\left(\mathbb{R}^{3}\right)} .
\end{aligned}
$$

Suppose that $\alpha<4$. If $(\alpha+4) / 8<1 / q \leq 1$ then $\alpha / 8-(2-q) /(2 q)<0$ and so for some $c>0$ and all $f \in S\left(\mathbb{R}^{3}\right)$,

$$
\sum_{k \geq k\left(\delta_{0}\right)}\|\widehat{f}\|_{L^{q}\left(\Sigma_{Q_{k}, \varphi}\right)} \leq c\|f\|_{L^{4 / 3}\left(\mathbb{R}^{3}\right)} .
$$

For $\delta>0$ and $j \in \mathbb{N} \cup\{0\}$, let

$$
A_{j, \delta}=\left\{\left(x_{1}, x_{2}\right) \in \mathbb{R}^{2}: 2^{-j-1} \leq\left|x_{1}\right| \leq 2^{-j},\left|x_{2}\right| \leq \delta\left|x_{1}\right|\right\},
$$


thus $V_{\delta}=\bigcup_{j \geq 0} A_{j, \delta}$. For $\delta$ small enough, (3.7) gives

$$
\|\widehat{f}\|_{L^{q}\left(\Sigma_{A_{0, \delta}, \varphi}\right)} \leq c\|f\|_{L^{4 / 3}\left(\mathbb{R}^{3}\right)} .
$$

If $m-2 \leq \alpha<4$ the condition $1 / q>(\alpha+4) / 8$ implies

$$
\frac{1}{q}>-\left(\frac{m}{2}+1\right) \frac{3}{4}+\frac{m}{2}+1
$$

and so $\left(\mathrm{i}^{\prime}\right)$ follows from Remark 2.1(d).

For $t \in[0,1]$ let $p_{t}$ be defined by $1 / p_{t}=3 t / 4+1-t$. For $k \geq k\left(\delta_{0}\right)$ and any $\alpha$, from (3.6) we get $\|\widehat{f}\|_{L^{1}\left(\Sigma_{Q_{k}, \varphi}\right)} \leq c_{4} 2^{k(\alpha / 8-1 / 2)}\|f\|_{L^{4 / 3}\left(\mathbb{R}^{3}\right)}$. Also $\|\widehat{f}\|_{L^{1}\left(\Sigma_{Q_{k}, \varphi}\right)} \leq c_{4} 2^{-k}\|f\|_{L^{1}\left(\mathbb{R}^{3}\right)}$, so an application of the Riesz-Thorin theorem gives

$$
\|\widehat{f}\|_{L^{1}\left(\Sigma_{Q_{k}, \varphi}\right)} \leq c 2^{k(((\alpha+4) / 8) t-1)}\|f\|_{L^{p_{t}\left(\mathbb{R}^{3}\right)}}
$$

for all $f \in S\left(\mathbb{R}^{3}\right)$. So, for $\delta$ small enough,

$$
\|\widehat{f}\|_{L^{1}\left(\Sigma_{A_{0, \delta}, \varphi}\right)} \leq \sum_{k \geq k\left(\delta_{0}\right)}\|\widehat{f}\|_{L^{1}\left(\Sigma_{Q_{k}, \varphi}\right)} \leq c\|f\|_{L^{p_{t}}\left(\mathbb{R}^{3}\right)}
$$

for all $t \in[0,8 /(\alpha+4))$ if $\alpha \geq 4$, and for all $t \in[0,1]$ if $\alpha<4$.

Suppose that $m-2 \leq \alpha<\infty$ and $\alpha \geq 4$. If $(\alpha+2) /(\alpha+4)<1 / p \leq 1$ then $1 / p=3 t / 4+1-t$ for some $t \in[0,8 /(\alpha+4))$. Also

$$
1>-\left(\frac{m}{2}+1\right) \frac{1}{p}+\frac{m}{2}+1 .
$$

So, Remark 2.1(d) and (3.9) imply $(1 / p, 1) \in E_{V_{\delta}, \varphi}$ and then (ii') holds.

Consider now the case $0 \leq \alpha<m-2, m \geq 6$ and suppose $m /(m+2)<$ $1 / p \leq 1$. A computation shows that $1 / p=1 / p_{t}$ for some $t \in[0,8 /(m+2))$ and so $t<8 /(\alpha+4)$. If $\alpha \geq 4$ then (3.9) and Remark 2.1(d) imply (iii') in this case. If $\alpha<4$, observe that the assumption on $p$ implies $3 / 4<1 / p$ and so (3.9) gives $(1 / p, 1) \in E_{A_{0, \delta}, \varphi}$. Since also

$$
1>-\left(\frac{m}{2}+1\right) \frac{1}{p}+\frac{m}{2}+1,
$$

(iii') follows in this case from Remark $2.1(\mathrm{~d})$.

Finally, assume that $0 \leq \alpha<m-2, m<6$ and $(m+2) / 8<1 / q \leq 1$. Then $(\alpha+4) / 8<1$, thus $(3.7)$ gives $(3 / 4,1 / q) \in E_{A_{0, \delta}, \varphi}$. Also $(m+2) / 8<$ $1 / q$ implies $1 / q>-(m / 2+1) 3 / 4+m / 2+1$ and so Remark $2.1(\mathrm{~d})$ gives $\left(\mathrm{iv}^{\prime}\right)$.

REMARK 3.2. For $\delta>0$ let

$$
W_{\delta}=\left\{x=\left(x_{1}, x_{2}\right) \in \mathbb{R}^{2}:|x| \leq 1,\left|x_{2}\right| \leq \delta\left|x_{1}\right|\right\}
$$

and let $V_{\delta}$ be defined by (3.1). Since $W_{\delta} \subset V_{\delta}$, it follows that Lemma 3.1 holds for $W_{\delta}$ in place of $V_{\delta}$. 
THEOREM 3.3. Let $\varphi: \mathbb{R}^{2} \rightarrow \mathbb{R}$ be a homogeneous polynomial function of degree $m \geq 2$ such that $\operatorname{det} \varphi^{\prime \prime}(x) \equiv 0$. Then for $m \geq 3$,

$$
E^{\circ}=\{(1 / p, 1 / q) \in[0,1] \times[0,1]: 1 / q>-(m+1) / p+m+1\},
$$

and for $m=2$,

$$
E^{\circ}=\{(1 / p, 1 / q) \in(3 / 4,1] \times[0,1]: 1 / q>-3 / p+3\} .
$$

Proof. From Remark 2.5 we have $\varphi\left(x_{1}, x_{2}\right)=\left(a x_{1}+b x_{2}\right)^{m}$ for some $a, b \in \mathbb{R}$ and all $\left(x_{1}, x_{2}\right) \in \mathbb{R}^{2}$, and so, by Remark 2.1(b), the problem reduces (after composing with a suitable rotation followed by a dilation) to the case $\varphi\left(x_{1}, x_{2}\right)=x_{2}^{m}$. From Remark 2.1(a), Lemma 2.7 and Remark 2.8, it suffices to see that for $m \geq 3$, if $m /(m+1)<1 / p \leq 1$ then $(1 / p, 1) \in E$, and for $m=2$, if $3 / 4<1 / p \leq 1$ and $1 / q>-3 / p+3$ then $(1 / p, 1 / q) \in E$. For $3 / 4<1 / p \leq 1$ we know that (see e.g. [3]) there exists $c>0$ such that

$$
\left(\int_{1 / 2 \leq|s| \leq 1}\left|\widehat{g}\left(s, s^{m}\right)\right|^{p} d s\right)^{1 / p} \leq c_{p}\|g\|_{L^{p}\left(\mathbb{R}^{2}\right)} \quad \text { for all } g \in S\left(\mathbb{R}^{2}\right) .
$$

We claim that for such $p$ there exists $c^{\prime}>0$ such that

$$
\left(\int_{\left|x_{1}\right| \leq 1} \int_{1 / 2 \leq\left|x_{2}\right| \leq 1}\left|\widehat{f}\left(x_{1}, x_{2}, x_{2}^{m}\right)\right|^{p} d x_{1} d x_{2}\right)^{1 / p} \leq c^{\prime}\|f\|_{L^{p}\left(\mathbb{R}^{3}\right)}
$$

for all $f \in S\left(\mathbb{R}^{3}\right)$. Indeed, for $h: \mathbb{R} \rightarrow \mathbb{C}$ and $g: \mathbb{R}^{2} \rightarrow \mathbb{C}$, let $h^{\wedge_{1}}, g^{\wedge_{2}}$ denote their one-and two-dimensional Fourier transforms respectively. Now,

$$
\begin{aligned}
& \left(\int_{\left|x_{1}\right| \leq 1} \int_{1 / 2 \leq\left|x_{2}\right| \leq 1}\left|\widehat{f}\left(x_{1}, x_{2}, x_{2}^{m}\right)\right|^{p} d x_{1} d x_{2}\right)^{1 / p} \\
& =\left\|\left(\int_{1 / 2 \leq\left|x_{2}\right| \leq 1}\left|\left(\left(\xi_{2}, \xi_{3}\right) \mapsto f\left(\cdot, \xi_{2}, \xi_{3}\right)^{\wedge 1}\left(x_{1}\right)\right)^{\wedge}\left(x_{2}, x_{2}^{m}\right)\right|^{p} d x_{2}\right)^{1 / p}\right\|_{X}
\end{aligned}
$$

where $X=L^{p}\left((-1,1), d x_{1}\right)$. From (3.10) we get

$$
\begin{aligned}
& \left(\int_{\left|x_{1}\right| \leq 1} \int_{1 / 2 \leq\left|x_{2}\right| \leq 1}\left|\widehat{f}\left(x_{1}, x_{2}, x_{2}^{m}\right)\right|^{p} d x_{1} d x_{2}\right)^{1 / p} \\
& \quad \leq c\left(\int_{\mathbb{R}^{2}}\left(\int_{\left|x_{1}\right| \leq 1}\left|f\left(\cdot, \xi_{2}, \xi_{3}\right)^{\wedge_{1}}\left(x_{1}\right)\right|^{p} d x_{1}\right) d \xi_{2} d \xi_{3}\right)^{1 / p} .
\end{aligned}
$$

Since $p<2$,

$$
\left\|f\left(\cdot, \xi_{2}, \xi_{3}\right)^{\wedge_{1}}\right\|_{L^{p}(-1,1)} \leq c^{\prime \prime}\left\|f\left(\cdot, \xi_{2}, \xi_{3}\right)^{\wedge_{1}}\right\|_{L^{p^{\prime}(-1,1)}} \leq c^{\prime \prime \prime}\left\|f\left(\cdot, \xi_{2}, \xi_{3}\right)\right\|_{L^{p}(\mathbb{R})}
$$

for some positive $c^{\prime \prime}$ and $c^{\prime \prime \prime}$. So (3.11) follows.

For $t>0, x=\left(x_{1}, x_{2}\right) \in \mathbb{R}^{2}, \xi=\left(\xi_{1}, \xi_{2}, \xi_{3}\right) \in \mathbb{R}^{3}$ let $t . x=\left(x_{1}, t x_{2}\right)$ and $t \circ \xi=\left(\xi_{1}, t \xi_{2}, t^{m} \xi_{3}\right)$. For $g: \mathbb{R}^{3} \rightarrow \mathbb{R}, t>0$ let $t \circ g: \mathbb{R}^{3} \rightarrow \mathbb{R}$ be defined by 
$(t \circ g)(\xi)=g(t \circ \xi)$. Finally, for $k \in \mathbb{N} \cup\{0\}$ let

$$
R_{k}=\left\{\left(x_{1}, x_{2}\right):\left|x_{1}\right| \leq 1,2^{-k-1} \leq\left|x_{2}\right| \leq 2^{-k}\right\} .
$$

So $R_{k}=2^{-k} R_{0}$ and from (3.11) a standard homogeneity argument gives

$$
\begin{aligned}
\|\widehat{f}\|_{L^{p}\left(\Sigma_{R_{k}, \varphi}\right)} & \leq c 2^{-k / p+k(m+1)}\left\|2^{k} \circ f\right\|_{L^{p}\left(\mathbb{R}^{3}\right)} \\
& =2^{-k / p+k(m+1)(1-1 / p)}\|f\|_{L^{p}\left(\mathbb{R}^{3}\right)}
\end{aligned}
$$

and so, by Hölder's inequality,

$$
\begin{aligned}
\|\widehat{f}\|_{L^{1}\left(\Sigma_{R_{k}, \varphi}\right)} & \leq 2^{-k / p+k(m+1)(1-1 / p)}\left|R_{k}\right|^{1-1 / p}\|f\|_{L^{p}\left(\mathbb{R}^{3}\right)} \\
& =2^{k(m-(m+1) / p)}\|f\|_{L^{p}\left(\mathbb{R}^{3}\right)} .
\end{aligned}
$$

In particular, if $m \geq 3$ and $m /(m+1)<1 / p \leq 1$ then $3 / 4<1 / p \leq 1$ and so from (3.14) we obtain

$$
\|\widehat{f}\|_{L^{1}(\Sigma)} \leq \sum_{k \geq 0}\|\widehat{f}\|_{L^{1}\left(\Sigma_{R_{k}, \varphi}\right)} \leq c\|f\|_{L^{p}\left(\mathbb{R}^{3}\right)}
$$

and the theorem follows for the case $m \geq 3$.

Consider now the case $m=2$. Suppose that $3 / 4<1 / p \leq 1$; then $-1 / p+$ $3(1-1 / p)<0$ and so from $(3.13)$,

$$
\|\widehat{f}\|_{L^{p}(\Sigma)} \leq \sum_{k \geq 0}\|\widehat{f}\|_{L^{p}\left(\Sigma_{R_{k}, \varphi}\right)} \leq c\|f\|_{L^{p}\left(\mathbb{R}^{3}\right)} .
$$

Also, Hölder's inequality gives $\|\widehat{f}\|_{L^{q}(\Sigma)} \leq c\|f\|_{L^{p}\left(\mathbb{R}^{3}\right)}$ for $1 / p \leq 1 / q \leq 1$. So the theorem follows from Remark 2.1(a).

THEOREM 3.4. Let $\varphi: \mathbb{R}^{2} \rightarrow \mathbb{R}$ be a homogeneous polynomial function of degree $m \geq 2$ such that $\operatorname{det} \varphi^{\prime \prime}(x)$ does not vanish identically, and let $\widetilde{m}$ be defined by (1.1) if $\operatorname{det} \varphi^{\prime \prime}(x)$ vanishes somewhere in $\mathbb{R}^{2}-\{0\}$, and by $\widetilde{m}=m$ if not. Then

(i) for $\widetilde{m} \geq 6$,

$$
E^{\circ}=\left\{(1 / p, 1 / q) \in[0,1] \times[0,1]: \frac{1}{q}>-\left(\frac{\widetilde{m}}{2}+1\right) \frac{1}{p}+\frac{\widetilde{m}}{2}+1\right\}
$$

(ii) for $\widetilde{m}<6$,

$$
\begin{aligned}
E^{\circ} \cap((3 / 4,1] \times[0,1]) & \\
& =\left\{(1 / p, 1 / q) \in(3 / 4,1] \times[0,1]: \frac{1}{q}>-\left(\frac{\widetilde{m}}{2}+1\right) \frac{1}{p}+\frac{\widetilde{m}}{2}+1\right\}
\end{aligned}
$$

and also $(3 / 4,1 / q) \in E$ for $(\widetilde{m}+2) / 8<1 / q \leq 1$.

Proof. To see that the stated conditions are sufficient, we consider first the case $\operatorname{det} \varphi^{\prime \prime}(x) \neq 0$ for all $x \in \mathbb{R}^{2}-\{0\}$. For $j \in \mathbb{N} \cup\{0\}$ let

$$
A_{j}=\left\{x \in \mathbb{R}^{2}: 2^{-j-1} \leq|x| \leq 2^{j}\right\} .
$$


From [8, p. 386] we have $(3 / 4,1 / 2) \in E_{A_{j}, \varphi}$ and so in this case the lemma follows from Remark 2.1(d).

Suppose now that $\operatorname{det} \varphi^{\prime \prime}(x)=0$ for some $x \in \mathbb{R}^{2}-\{0\}$. Let $L_{1}, \ldots, L_{k}$ be as in the introduction. For $\delta>0$ let

$$
C_{\delta}^{j}=\left\{x \in B:\left|\pi_{L_{j}^{\perp}}(x)\right| \leq \delta\left|\pi_{L_{j}}(x)\right|\right\}
$$

where $\pi_{L_{j}}$ and $\pi_{L_{j}^{\perp}}$ denote the orthogonal projections from $\mathbb{R}^{2}$ onto $L_{j}$ and $L_{j}^{\perp}$ respectively. Choose $\delta$ small enough such that $C_{\delta}^{i} \cap C_{\delta}^{j}=\emptyset$ for $i \neq j$. So $\operatorname{det} \varphi^{\prime \prime}(x) \neq 0$ for $x \in C_{\delta}^{j}-L_{j}$. Let $T_{j} \in \mathrm{SO}(2)$ be such that $T_{j}\left(L_{j}\right)$ is the $x_{1}$ axis, let $\psi_{j}=\varphi \circ T_{j}^{-1}, \widetilde{C}_{\delta}^{j}=T_{j}\left(C_{\delta}^{j}\right)$ and let $\widetilde{\alpha}_{j}$ be the vanishing order of $x_{2} \mapsto \operatorname{det} \psi_{j}^{\prime \prime}\left(1, x_{2}\right)$. Since the curvature is invariant under rotations we have $\widetilde{\alpha}_{j}=\alpha_{j}, j=1, \ldots, k$. We also have $E_{C_{\delta}^{j}, \varphi}=E_{T_{j}\left(C_{\delta}\right), \varphi \circ T_{j}^{-1}}$ (see Remark 2.1(b)). Let $C_{\delta}=\bigcup_{1 \leq j \leq k} C_{\delta}^{j}$, so $E_{C_{\delta}, \varphi}=\bigcap_{1 \leq j \leq k} E_{C_{\delta}^{j}, \varphi}$.

Suppose that $(1 / p, 1 / q)$ belongs to the left side of the equality in either (i) or (ii). If $\widetilde{m}=m$, Remark 2.1 (d) applies. If $\widetilde{m}=\alpha_{j}+2$ for some $j=1, \ldots, k$, we apply Lemmas 2.4 and 2.7 to $\varphi \circ T_{j}^{-1}$ to deduce that $(1 / p, 1 / q)$ belongs to the right side of the equality.

On the other hand, from Lemma 3.1 and Remark 3.2 it follows that, for $\delta$ positive and small enough, if $\widetilde{m}<6$,

$$
\frac{3}{4} \leq \frac{1}{p} \leq 1 \quad \text { and } \quad-\frac{\widetilde{m}+2}{2 p}+\frac{\widetilde{m}+2}{2}<\frac{1}{q} \leq 1
$$

then $(1 / p, 1 / q) \in E_{C_{\delta}, \varphi}$, and if $\widetilde{m} \geq 6$,

$$
\frac{\widetilde{m}}{\widetilde{m}+2}<\frac{1}{p} \leq 1 \quad \text { and } \quad-\frac{\widetilde{m}+2}{2 p}+\frac{\widetilde{m}+2}{2}<\frac{1}{q} \leq 1
$$

then $(1 / p, 1 / q) \in E_{C_{\delta}, \varphi}$. Finally, let $D_{\delta}=B-C_{\delta}$. So $D_{\delta}$ is a union of a finite number of angular sectors with vertices at the origin where $\operatorname{det} \varphi^{\prime \prime}(x)$ never vanishes (except at the origin), and so we can proceed as in the first part of the proof to get $(1 / p, 1 / q) \in E_{D_{\delta}, \varphi}$ for

$$
\frac{3}{4} \leq \frac{1}{p} \leq 1 \quad \text { and } \quad-\frac{\widetilde{m}+2}{2 p}+\frac{\widetilde{m}+2}{2}<\frac{1}{q} \leq 1 .
$$

Since $E=E_{C_{\delta}, \varphi} \cap E_{D_{\delta}, \varphi}$ the theorem follows.

\section{Sharp $L^{p}\left(\mathbb{R}^{3}\right)-L^{2}(\Sigma)$ estimates for $\mathcal{R}$}

REMARK 4.1. For our next results we will need to introduce two Littlewood-Paley decompositions on $S\left(\mathbb{R}^{3}\right)$. Let $\Phi \in C_{\mathrm{c}}^{\infty}(\mathbb{R})$ be an even function satisfying $0 \leq \Phi \leq 1, \operatorname{supp} \Phi \subset\left\{t \in \mathbb{R}: 2^{-1} \leq|t| \leq 2\right\}$ and such that $\sum_{r \in \mathbb{Z}} \Phi\left(2^{r} t\right)=1$ if $t \neq 0$. For $r \in \mathbb{Z}$ let $\Psi_{r}: \mathbb{R} \rightarrow \mathbb{R}$ be defined by $\Psi_{r}(t)=$ $2^{-r} \widehat{\Phi}\left(2^{-r} t\right)$, and for $f \in S\left(\mathbb{R}^{3}\right)$ let $T_{r} f$ be given by $T_{r} f=\left(\delta \otimes \Psi_{r} \otimes \delta\right) * f$. 
Thus $f=\sum_{r \in \mathbb{Z}} T_{r} f$ with convergence in $S^{\prime}\left(\mathbb{R}^{3}\right)$. Moreover, it can be checked that for $\varepsilon_{r}= \pm 1$, the one-dimensional multiplier $\sum_{r \in \mathbb{Z}} \varepsilon_{r} \Phi\left(2^{r} t\right)$ satisfies the hypothesis of Theorem 3 on p. 96 of [7] with constants independent of the choice of $\varepsilon_{r}$. Hence for $f \in S\left(\mathbb{R}^{3}\right),\left\|\sum_{r \in \mathbb{Z}} \varepsilon_{r} T_{r} f\right\|_{L^{p}\left(\mathbb{R}^{3}\right)} \leq c\|f\|_{L^{p}\left(\mathbb{R}^{3}\right)}$ with $c$ independent of $f$ and of the choice of $\varepsilon_{r}$, so as in [7, p. 105] we have the Littlewood-Paley inequality

$$
\left\|\left(\sum_{r \in \mathbb{Z}}\left|T_{r} f\right|^{2}\right)^{1 / 2}\right\|_{L^{p}\left(\mathbb{R}^{3}\right)} \leq c\|f\|_{L^{p}\left(\mathbb{R}^{3}\right)} .
$$

Similarly, if we start with an even function $\widetilde{\Phi} \in C_{\mathrm{c}}^{\infty}\left(\mathbb{R}^{2}\right)$ with support contained in the annulus $\left\{t \in \mathbb{R}^{2}: 2^{-1} \leq|t| \leq 2\right\}$ such that $0 \leq \widetilde{\Phi} \leq 1$, $\sum_{r \in \mathbb{Z}} \widetilde{\Phi}\left(2^{r} t\right)=1$ for $t \in \mathbb{R}^{2}-\{0\}$, and if we define $\widetilde{\Psi}_{r}(t)=2^{-2 r}(\widetilde{\Phi})^{\wedge}\left(2^{-r} t\right)$, $r \in \mathbb{Z}$, and $\widetilde{T}_{r} f=\left(\widetilde{\Psi}_{r} \otimes \delta\right) * f$, we now see that $f=\sum_{r \in \mathbb{T}} \widetilde{T}_{r} f, f \in S\left(\mathbb{R}^{3}\right)$, with convergence in $S^{\prime}\left(\mathbb{R}^{3}\right)$ and that (4.1) is also true for the family $\left\{\widetilde{T}_{r}\right\}_{r \in \mathbb{Z}}$ in place of $\left\{T_{r}\right\}_{r \in \mathbb{Z}}$.

THEOREM 4.2. Let $\varphi: \mathbb{R}^{2} \rightarrow \mathbb{R}$ be a homogeneous polynomial function of degree $m \geq 2$ such that $\operatorname{det} \varphi^{\prime \prime}(x) \equiv 0$. Then $((2 m+1) /(2 m+2), 1 / 2) \in E$.

Proof. As in Theorem 3.3 it is enough to prove the theorem for the case $\varphi(x)=x_{2}^{m}$. In this case the van der Corput lemma applied with the $m$ th derivative gives

$$
\left|\int_{-1}^{1} e^{-i\left(y \xi_{2}+y^{m} \xi_{3}\right)} d y\right| \leq c\left|\xi_{3}\right|^{-1 / m},
$$

so $\left|\sigma^{\wedge}(\xi)\right| \leq c\left(1+\left|\xi_{3}\right|\right)^{-1 / m}$. Thus the theorem follows from Remark 2.6.

Lemma 4.3. Let $\varphi: \mathbb{R}^{2} \rightarrow \mathbb{R}$ be a homogeneous polynomial function such that $\operatorname{det} \varphi^{\prime \prime}(x)$ is not identically zero, let $m^{*}$ be as in Lemma 3.1 and for $\delta>0$ let $V_{\delta}$ be defined by (3.1). Then for $\delta$ positive and small enough, $\left(\left(m^{*}+1\right) /\left(m^{*}+2\right), 1 / 2\right) \in E_{V_{\delta, \varphi}}$.

Proof. We first consider the case $\alpha<m-2$. Then $m^{*}=m$. Let $\delta_{0}$ and $k\left(\delta_{0}\right)$ be as in the proof of Lemma 3.1 and for $k \in \mathbb{Z} \cup\{0\}$ let $Q_{k}$ be defined by (3.3). For $\theta \in[0,1]$ let $p_{\theta}$ be defined by $1 / p_{\theta}=3 \theta / 4+1-\theta$. For $f \in S\left(\mathbb{R}^{3}\right)$, from (3.5) and $\|\widehat{f}\|_{L^{2}\left(\Sigma_{Q_{k}, \varphi}\right)} \leq c 2^{-k / 2}\|f\|_{L^{1}\left(\mathbb{R}^{3}\right)}$ (see Remark 2.1(a)), the Riesz-Thorin theorem gives

$$
\|\widehat{f}\|_{L^{2}\left(\Sigma_{Q_{k}, \varphi}\right)} \leq c 2^{k \alpha \theta / 8-k(1-\theta) / 2}\|f\|_{L^{p_{\theta}\left(\mathbb{R}^{3}\right)}} .
$$

Then for $\delta$ positive and small enough we get

$$
\|\widehat{f}\|_{L^{2}\left(\Sigma_{A_{0, \delta}, \varphi}\right)} \leq \sum_{k \geq k\left(\delta_{0}\right)}\|\widehat{f}\|_{L^{2}\left(\Sigma_{\left.Q_{k}, \varphi\right)}\right.} \leq c\|f\|_{L^{(m+2) /(m+1)\left(\mathbb{R}^{3}\right)}}
$$


with $A_{0, \delta}$ defined by (3.8). Thus, from Remark 2.1 (c) we find

$$
\|\widehat{f}\|_{L^{2}\left(\Sigma_{A_{j, \delta}, \varphi}\right)} \leq c\|f\|_{L^{(m+2) /(m+1)\left(\mathbb{R}^{3}\right)}}
$$

for some $c>0$ and all $j \in \mathbb{Z}, f \in S\left(\mathbb{R}^{3}\right)$.

For $r \in \mathbb{Z}$ let $\widetilde{T}_{r}, \widetilde{\Psi}_{r}$ be as in Remark 4.1. Then for $f \in S\left(\mathbb{R}^{3}\right)$ and $\xi=\left(\xi_{1}, \xi_{2}, \xi_{3}\right)$ such that $\left(\xi_{1}, \xi_{2}\right) \neq 0$ we have

$$
\widehat{f}(\xi)=\sum_{r \in \mathbb{Z}} \widehat{\widetilde{\Psi}}_{r}\left(\xi_{1}, \xi_{2}\right) \widehat{f}(\xi)=\sum_{r \in \mathbb{Z}}\left(\widetilde{T}_{r} f\right)^{\wedge}(\xi)
$$

with convergence in $L^{2}\left(\mathbb{R}^{3}\right)$. Also, $\left(\widetilde{T}_{r} f\right)^{\wedge}(\xi)=\widetilde{\Phi}\left(2^{r}\left(\xi_{1}, \xi_{2}\right)\right) \widehat{f}(\xi)$ and so, for each $\xi$, the set $\left\{r \in \mathbb{Z}:\left(\widetilde{T}_{r} f\right)^{\wedge}(\xi) \neq 0\right\}$ has at most three elements. Then

$$
\left|\sum_{r \in \mathbb{Z}}\left(\widetilde{T}_{r} f\right)^{\wedge}(x, \varphi(x))\right|^{2} \leq 3 \sum_{r \in \mathbb{Z}}\left|\left(\widetilde{T}_{r} f\right)^{\wedge}(x, \varphi(x))\right|^{2} .
$$

Let $A_{r, \delta}^{\prime}=\bigcup_{j=r-1}^{r+1} A_{j, \delta}$. From (4.3) we have

$$
\int_{A_{r, \delta}^{\prime}}\left|\left(\widetilde{T}_{r} f\right)^{\wedge}(x, \varphi(x))\right|^{2} d x \leq c\left\|\widetilde{T}_{r} f\right\|_{L^{(m+2) /(m+1)}\left(\mathbb{R}^{3}\right)}^{2}
$$

with $c$ independent of $f$ and $r$. Also, if $0<\delta<1$ then

$$
\left\{x \in V_{\delta}:\left(\widetilde{T}_{r} f\right)^{\wedge}(x, \varphi(x)) \neq 0\right\} \subset A_{r, \delta}^{\prime} .
$$

From these facts we deduce that there exist positive constants $c, c^{\prime}$ and $c^{\prime \prime}$ independent of $f$ such that

$$
\begin{aligned}
\|\widehat{f}\|_{L^{2}\left(\Sigma_{V_{\delta}, \varphi}\right)}^{2} & =\int_{V_{\delta}}\left|\sum_{r \in \mathbb{Z}}\left(\widetilde{T}_{r} f\right)^{\wedge}(x, \varphi(x))\right|^{2} d x \\
& \leq 3 \sum_{r \in \mathbb{Z}} \int_{A_{r, \delta}^{\prime}}\left|\left(\widetilde{T}_{r} f\right)^{\wedge}(x, \varphi(x))\right|^{2} d x \leq c \sum_{r \in \mathbb{Z}}\left\|\widetilde{T}_{r} f\right\|_{L^{(m+2) /(m+1)}\left(\mathbb{R}^{3}\right)}^{2} \\
& \leq c^{\prime}\left\|\left(\sum_{r \in \mathbb{Z}}\left|\widetilde{T}_{r} f\right|^{2}\right)^{1 / 2}\right\|_{L^{(m+2) /(m+1)\left(\mathbb{R}^{3}\right)}} \leq c^{\prime \prime}\|f\|_{L^{(m+2) /(m+1)}\left(\mathbb{R}^{3}\right)}^{2}
\end{aligned}
$$

where the last inequality follows from the Littlewood-Paley inequality and the previous one from Minkowski's inequality. Thus the lemma follows for $\alpha<m-2$.

If $\alpha \geq m-2$ then $m^{*}=\alpha+2$ and so from (4.2) we obtain $\|\widehat{f}\|_{L^{2}\left(\Sigma_{Q_{k}, \varphi}\right)} \leq$ $c\|f\|_{L^{\left(m^{*}+2\right) /\left(m^{*}+1\right)\left(\mathbb{R}^{3}\right)}}$ for some $c>0$, for all $k \geq k\left(\delta_{0}\right)$ and $f \in S\left(\mathbb{R}^{3}\right)$.

For $r \in \mathbb{Z}$ let $T_{r}, \Psi_{r}$ be as in Remark 4.1. Then for $f \in S\left(\mathbb{R}^{3}\right)$ and $\xi=\left(\xi_{1}, \xi_{2}, \xi_{3}\right)$ such that $\xi_{2} \neq 0$ we have

$$
\widehat{f}(\xi)=\sum_{r \in \mathbb{Z}} \widehat{\Psi}_{r}\left(\xi_{2}\right) \widehat{f}(\xi)=\sum_{r \in \mathbb{Z}}\left(T_{r} f\right)^{\wedge}(\xi)
$$


with convergence in $L^{2}\left(\mathbb{R}^{3}\right)$. Now we proceed as in the first part of the proof, but with $\widetilde{T}_{r}$ replaced by $T_{r}$, to obtain

$$
\|\widehat{f}\|_{L^{2}\left(\Sigma_{A_{0, \delta}, \varphi}\right)}^{2} \leq c\|f\|_{L^{\left(m^{*}+2\right) /\left(m^{*}+1\right)\left(\mathbb{R}^{3}\right)}}^{2} .
$$

From Remark 2.1(c) we get

$$
\begin{aligned}
& \|\widehat{f}\|_{L^{2}\left(\Sigma_{A_{j, \delta}, \varphi}\right)} \\
& \leq c 2^{-j\left[1+(m+2)\left(m^{*}+1\right) /\left(m^{*}+2\right)-(m+2)\right]}\|f\|_{L^{\left(m^{*}+2\right) /\left(m^{*}+1\right)}\left(\mathbb{R}^{3}\right)} \\
& =c 2^{-j\left(m^{*}-m\right) /\left(m^{*}+2\right)}\|f\|_{L^{\left(m^{*}+2\right) /\left(m^{*}+1\right)}\left(\mathbb{R}^{3}\right)} .
\end{aligned}
$$

If $\alpha>m-2$ then $m^{*}>m$, so we can perform the sum on $j$ to obtain the conclusion.

Finally, for $\alpha=m-2$ the estimates in (4) are uniform on $j$ and then we proceed as in the case $\alpha<m-2$ to get the assertion.

From this lemma we proceed as in the proof of Theorem 3.4 to obtain the following

THEOREM 4.4. Let $\varphi: \mathbb{R}^{2} \rightarrow \mathbb{R}$ be a homogeneous polynomial function such that $\operatorname{det} \varphi^{\prime \prime}(x)$ is not identically zero, and let $\widetilde{m}$ be as in Theorem 3.4 . Then $((\widetilde{m}+1) /(\widetilde{m}+2), 1 / 2) \in E$.

\section{References}

[1] M. Christ, On the restriction of the Fourier transform to curves: endpoint results and the degenerate case, Trans. Amer. Math. Soc. 287 (1985), 223-238.

[2] M. Dellanegra, Problemi di restrizione della trasformata di Fourier ad alcune ipersuperfici di $\mathbb{R}^{n}$, Ph.D. thesis, Politecnico di Torino, 1998.

[3] C. Fefferman, Inequalities for strongly singular convolution operators, Acta Math. 124 (1970), 9-36.

[4] E. Prestini, Restriction theorems for the Fourier transform to some manifolds in $\mathbb{R}^{n}$, in: Proc. Sympos. Pure Math. 35, Part 1, Amer. Math. Soc., Providence, RI, 1979, 101-109.

[5] P. Sjölin, Fourier multipliers and estimates of the Fourier transform of measures carried by smooth curves in $\mathbb{R}^{2}$, Studia Math. 51 (1974), 169-182.

[6] C. D. Sogge, A sharp restriction theorem for degenerated curves in $\mathbb{R}^{2}$, Amer. J. Math. 109 (1987), 223-228.

[7] E. M. Stein, Singular Integrals and Differentiability Properties of Functions, Princeton Univ. Press, Princeton, NJ, 1970.

[8] -, Harmonic Analysis, Real-Variable Methods, Orthogonality, and Oscillatory Integrals, Princeton Univ. Press, Princeton, NJ, 1993.

[9] E. Stein and G. Weiss, Introduction to Fourier Analysis on Euclidean Spaces, Princeton Univ. Press, Princeton, NJ, 1971.

[10] R. S. Strichartz, Restrictions of Fourier transforms to quadratic surfaces and decay of solutions of wave equations, Duke Math. J. 44 (1977), 705-713. 
[11] P. A. Tomas, Restriction theorems for the Fourier transform, in: Proc. Sympos. Pure Math. 35, Part 1, Amer. Math. Soc., Providence, RI, 1979, 111-114.

FaMAF, Universidad Nacional de Córdoba

and CIEM-CONICET

Ciudad Universitaria

5000 Córdoba, Argentina

E-mail: eferrey@mate.uncor.edu

godoy@mate.uncor.edu

urciuolo@mate.uncor.edu

Received May 6, 2002 